\title{
Inverse Evaluation of Material Constants for Composite Plates by Optical Interferometry Method
}

\author{
Chien-Ching $\mathrm{Ma}^{*}$ and Chan-Chiao Lin ${ }^{\dagger}$ \\ National Taiwan University, Taipei 10617, Taiwan, Republic of China
}

\begin{abstract}
Electronic speckle pattern interferometry (ESPI) is a powerful tool for the full field, noncontact, and real-time measurement method of surface displacements for deformed bodies. As compared with the conventional film recording and optical reconstruction procedures used for holographic interferometry, the interferometric fringes of ESPI are produced instantly by a video recording system. In this study, the amplitudefluctuation ESPI (AF-ESPI) optical system is used for measuring the resonant frequencies and mode shapes of vibrating composite plates. By making use of the resonant frequencies for free plates obtained experimentally, an inverse evaluationfor the material constants is developed using the Rayleigh-Ritz technique and the Simplex method for function minimization. Finally, finite element calculation is used to construct the mode shapes from the obtained material constants by inverse evaluation, and the results are compared with the experimental observations. The experimental method based on optical AF-ESPI setup and the inverse algorithms proposed might become a reliable and self-consistent methodology for evaluating material constants of composite plates.
\end{abstract}

\begin{tabular}{ll} 
& \multicolumn{1}{c}{ Nomenclature } \\
$A$ & $=$ vibration amplitude \\
$D_{i j}$ & $=$ laminate bending stiffness \\
$I_{A}$ & $=$ object light intensity \\
$I_{B}$ & $=$ reference light intensity \\
$w$ & $=$ transverse displacement \\
$\theta$ & $=$ angle between object light and observation direction \\
$\lambda$ & $=$ wavelength of laser \\
$\rho$ & $=$ mass density \\
$\tau$ & $=$ charge-coupled device refreshing time \\
$\phi$ & $=$ phase difference between object and reference light \\
$\omega$ & $=$ vibration frequency
\end{tabular}

\section{Introduction}

$\mathbf{H}$ OLOGRAPHIC interferometry opened new worlds of research by making possible accurate, global measurement of small dynamic surface displacements in a two-step process for a wide variety of objects. For this purpose, different methods of holographic interferometry have been developed for vibration analysis, which have made possible the gathering of a large amount of practical and theoretical information. Unfortunately, the slow and cumbersome process of film development limits the application of holographic vibration analysis in industry. Electronic speckle pattern interferometry (ESPI) was proposed ${ }^{1}$ in the 1970 s as a method of producing the interferogram without using traditional holographic technique. ${ }^{2}$ The main difference between ESPI and holography is the interferometric image processing. The image data are digitized by video camera and digital signal processor for the ESPI method, which will eliminate the time-consuming chemical development and will speed up the process. Because the interferometric image is recorded and updated by the video camera every $\frac{1}{30} \mathrm{~s}$, ESPI is faster to operate and more insensitive to the environment than holography. However, this method cannot reach the high image quality of holographic interferometry due to the low resolution of the video camera system. For practical applications, these disadvantages are outweighed by the high sampling rate of the video camera. For these reasons, ESPI has become a powerful technique in academic research and engineering applications. Because ESPI uses video

Received 26 September 1998; revision received 5 February 1999; accepted for publication 6 February 1999. Copyright (C) 1999 by the American Institute of Aeronautics and Astronautics, Inc. All rights reserved.

* Professor, Department of Mechanical Engineering.

${ }^{\dagger}$ Graduate Student, Department of Mechanical Engineering. recording and display, it works in real time to measure dynamic displacement. Its real-time nature makes it possible to implement this technique for vibration measurement. The most widely used experimental setup to study vibration by ESPI is the time-averaged method. ${ }^{3}$ The disadvantage of this method is that the interferometric fringes represent the amplitude but not the phase of the vibration. Løkberg and Hogmoen ${ }^{4}$ developed the phase-modulation method, which used the reference beam modulation technique to determine the relative phase of the vibration object. To reduce the noise coming from environment, the subtraction method was developed., ${ }^{5}$ The subtraction method differs from the time-averaged method in that the reference frame is first recorded before vibration and continuously subtracted from the incoming frames after vibration. However, the interferometric fringe visibility of the subtraction method is not good enough for quantitativemeasurement. To increase the visibility of the fringe pattern and to reduce the environmental noise simultaneously, an amplitude-fluctuation ESPI method was proposed by Wang et al. ${ }^{7}$ for out-of-plane vibration measurement. In the amplitude fluctuation ESPI (AF-ESPI) method, the reference frame is recorded in a vibrating state and subtracted from the incoming frame. Consequently, it combines the advantages of the timeaveraged and subtraction methods, i.e., good visibility and noise reduction. Ma and Huang ${ }^{8}$ and Huang and $\mathrm{Ma}^{9}$ used the AF-ESPI method to investigate the three-dimensional vibrations of piezoelectric rectangular parallelepipeds and cylinders; both the resonant frequencies and mode shapes were presented and discussed in detail.

Modern engineering design requires the use of material in a way that optimizes their inherent properties. The general class of materials that is most suitable for optimum design is composites. Composite materials are used in engineering structures for a variety of reasons, such as high specific stiffness. Composite structural elements are now used in a variety of components for automotive, aerospace, marine, and architectural structures, in addition to consumer products. Structures made of advanced composites, such as fiber-reinforced plastics, often consist of a number of layers having unidirectional fibers. These layers, or laminas, will generally be orthotropic. When orthotropic laminas are stacked to form a laminate, the resulting structure is generally anisotropic. The laminate will be orthotropic only for certain stacking sequences. The micromechanics is concerned with the mechanical behavior of constituent materials, the interaction of these constituents, and the resulting behavior of the basic composite. However, the macromechanics is concerned with the gross mechanical behavior of composite materials and structures without regard for the constituentmaterials or their interactions. This macromechanical behavior may be characterized 
by averaged stresses and strains and effective mechanical properties in an equivalent homogeneous material.

The measurement of mechanical properties is an important element of the quality control and quality assurance processes associated with the manufacture of composite materials. Because of the special characteristics of composites, the measurement of stiffnesses for composite materials is more difficult than for isotropic measurement. This is mainly due to the number of independent coefficients to be determined and the coupling effects; it has been found that the mechanical test methods that are used for conventional metallic materials are usually not applicable to composites. Thus, the development and evaluation of new test methods for composites have been major challenges for the experimental mechanics community. In the general case, a thin symmetric composite plate is characterized by 12 independent parameters: 6 in-plane and 6 bending stiffnesses. Standard tests are available to determine these parameters, but several samples are necessary, which lead these tests to be destructive. The American Society for Testing and Materials Standards D3039-76 and D4255-83 present static tensile and shear tests for determining the elastic constants of fiber-reinforced composites. To obtain one set of constants, two tensile test specimens and one shear test specimen must be fabricated and tested. The disadvantages of these tests are that resulting strains measured by strain gauges occur at localized areas and that they are slow, expensive, and destructive tests.

If vibrations are induced in a composite plate, then its dynamic response will be a function of plate geometry, density, boundary conditions, and the elastic constants. This implies the possibility of using plate vibration theory to develop a non-destructive test to determine the dynamic elastic constants of a composite plate. References 10 and 11 each presented a unique method for determining the elastic constants of an isotropic plate. Reference 12 reported a technique for determining the Poisson ratio of an orthotropic plate. The Rayleigh-Ritz or Galerkin techniques are often used to obtain approximate solutions for the mode shapes and natural frequencies. This approach has been investigated in many papers, in which the discussion is focused mainly on the iterative procedure providing elastic constants from plate vibration measurements and on the type of displacementfunctions used. For instance, Deobald and Gibson ${ }^{13}$ determined elastic constants of orthotropic plates with boundary conditions consisting of clamped and free edges.

In this paper, we employ an optical method based on the AF-ESPI to study the resonant properties of composite plates. The advantage of using the AF-ESPI method is that resonant frequencies and the corresponding mode shapes can be obtained simultaneously from the experimental measurement. By making use of the resonant frequencies for free composite plates obtained experimentally, an inverse evaluation for the material constants is investigated. Four elastic constants, namely, $E_{x}, E_{y}, G_{x y}$, and $v_{x y}$ are determined, and satisfactory results are obtained. Finally, finite element calculation is used to construct the mode shapes, and the results are compared with the experimental measurements. It is suggested that the experimental method based on the optical AF-ESPI setup and the inverse technique proposed in this study might become an applicable way to evaluate material constants for composite materials.

\section{Theory of AF-ESPI Method for Out-of-Plane Vibration Measurement}

The optical arrangement of ESPI method for out-of-plane vibrating measurement is shown schematically in Fig. 1. If the image of the specimen is taken at the stress free state, the light intensity detected by a charge-coupled device (CCD) camera can be expressed by the time-averaged method as

$$
\begin{aligned}
I_{0} & =\frac{1}{\tau} \int_{0}^{\tau}\left(I_{A}+I_{B}+2 \sqrt{I_{A} I_{B}} \cos \phi\right) \mathrm{d} t \\
& =I_{A}+I_{B}+2 \sqrt{I_{A} I_{B}} \cos \phi
\end{aligned}
$$

Assuming the specimen vibrates at a resonant frequency, then the light intensity taken by a CCD camera can be represented as

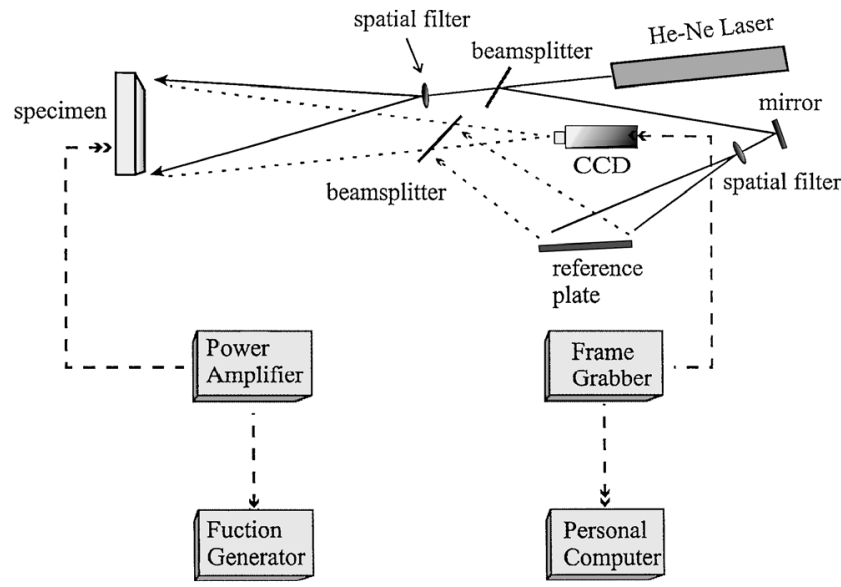

Fig. 1 Schematic of ESPI setup for out-of-plane measurement.

$$
\begin{aligned}
I_{1} & =\frac{1}{\tau} \int_{0}^{\tau}\left\{I_{A}+I_{B}+2 \sqrt{I_{A} I_{B}}\right. \\
& \left.\times \cos \left[\phi+\frac{2 \pi}{\lambda}(1+\cos \theta) A \cos \omega t\right]\right\} \mathrm{d} t
\end{aligned}
$$

Let $\Gamma=(2 \pi / \lambda)(1+\cos \theta)$ and assume $\tau=2 m \pi / \omega$, where $m$ is an integer; then Eq. (2) can be expressed as

$$
I_{1}=I_{A}+I_{B}+2 \sqrt{I_{A} I_{B}}(\cos \phi) J_{0}(\Gamma A)
$$

When these two images $\left(I_{0}\right.$ and $\left.I_{1}\right)$ are subtracted and rectified by the image processing system, i.e., Eq. (1) is subtracted from Eq. (3), the resulting image intensity can be expressed as

$$
I=I_{1}-I_{0}=2 \sqrt{I_{A} I_{B}}\left|(\cos \phi)\left[J_{0}(\Gamma A)-1\right]\right|
$$

The light intensity of the image shown in Eq. (4) is called the $s u b$ traction method, and the reference image is recorded before loading is applied.

Instead of using subtraction method, the AF-ESPI method is employed in this study by taking two images after the specimen vibrates and assuming that the vibration amplitude of the second image has changed from $A$ to $A+\Delta A$ due to the instability of the apparatus. The light intensity of the second image will be

$$
I_{2}=\frac{1}{\tau} \int_{0}^{\tau}\left\{I_{A}+I_{B}+2 \sqrt{I_{A} I_{B}} \cos [\phi+\Gamma(A+\Delta A) \cos \omega t]\right\} \mathrm{d} t
$$

Expand Eq. (5) by using a Taylor series and neglect higher-order terms; we then have

$$
I_{2}=I_{A}+I_{B}+2 \sqrt{I_{A} I_{B}}(\cos \phi)\left[1-\frac{1}{4} \Gamma^{2}(\Delta A)^{2}\right] J_{0}(\Gamma A)
$$

When these two images $\left(I_{1}\right.$ and $\left.I_{2}\right)$ are subtracted and rectified by the image processing system, i.e., Eq. (3) is subtracted from Eq. (6), the resulting image intensity can be expressed as

$$
I=I_{2}-I_{1}=\frac{\sqrt{I_{A} I_{B}}}{2}\left|(\cos \phi) \Gamma^{2}(\Delta A)^{2} J_{0}(\Gamma A)\right|
$$

Compared with the subtraction method where the reference image is taken at stress free state, the reference image is recorded and subtracted at the vibrating state by the AF-ESPI method. From Eqs. (4) and (7), the dominant function of the AF-ESPI method is $\left|J_{0}(\Gamma A)\right|$, and that of the subtraction method is $\left|J_{0}(\Gamma A)-1\right|$. Because of the discrepancy between the dominant functions, the nodal lines of vibrating interferometric patterns obtained by AF-ESPI method are the brightness lines and those by subtraction method are the darkness lines. This characteristic of the AF-ESPI method can be used as a qualitative observation or quantitative analysis for 
the fringe patterns. Furthermore, the sensitivity and the fringe visibility of the AF-ESPI method are better than those of the subtraction method. It can be verified that the number of fringes presented by the AF- ESPI method is about twice that by the subtraction method under the assumption of the same vibration amplitude. In addition to the theory of out-of-plane measurement already mentioned, the in-plane vibration measurement by AF-ESPI method also can be derived in a similar way. ${ }^{8}$

\section{Vibration of Composite Plates}

For a symmetric laminate plate subjected to bending only, the laminate moment-curvature relationships are given by

$$
\left\{\begin{array}{l}
M_{x} \\
M_{y} \\
M_{x y}
\end{array}\right\}=\left[\begin{array}{lll}
D_{11} & D_{12} & D_{16} \\
D_{12} & D_{22} & D_{26} \\
D_{16} & D_{26} & D_{66}
\end{array}\right]\left\{\begin{array}{c}
k_{x} \\
k_{y} \\
k_{x y}
\end{array}\right\}
$$

It is sometimes more convenient to use effective laminate engineering constants rather than the laminate stiffnesses. These effective laminate engineering constants can be derived by using laminate compliances. The results are expressed by the following equations:

$$
\begin{aligned}
E_{x} & =\frac{12 \operatorname{det}(D)}{\left(D_{22} D_{66}-D_{26}^{2}\right) h^{3}} \\
E_{y} & =\frac{12 \operatorname{det}(D)}{\left(D_{11} D_{66}-D_{16}^{2}\right) h^{3}} \\
G_{x y} & =\frac{12 \operatorname{det}(D)}{\left(D_{11} D_{22}-D_{12}^{2}\right) h^{3}} \\
v_{x y} & =\frac{D_{12} D_{66}-D_{16} D_{26}}{\left(D_{22} D_{66}-D_{26}^{2}\right)}
\end{aligned}
$$

where $h$ is the laminate thickness and $\operatorname{det}(D)$ is the determinant of the bending stiffness matrix. Although the laminate stiffness are meaningful parameters for all laminate configurations, the engineering constants may not be. The use of effective engineering constants must be restricted to those cases where the deformations are similar to the deformations associated with the engineering constant being used.

The partial differential equation governing the transverse motion of a symmetrically laminated thin plate is

$$
\begin{gathered}
D_{11} \frac{\partial^{4} w}{\partial x^{4}}+4 D_{16} \frac{\partial^{4} w}{\partial x^{3} \partial y}+2\left(D_{12}+D_{66}\right) \frac{\partial^{4} w}{\partial x^{2} \partial y^{2}} \\
+4 D_{26} \frac{\partial^{4} w}{\partial x \partial y^{3}}+D_{22} \frac{\partial^{4} w}{\partial y^{4}}=-\rho h \frac{\partial^{2} w}{\partial t^{2}}
\end{gathered}
$$

For the orthotropicplate, we have $D_{16}=D_{26}=0$. For free harmonic vibrations at frequency $\omega$, we can assume that

$$
w(x, y, t)=W(x, y) \sin \omega t
$$

where $W(x, y)$ is the maximum deflection and can be represented as a linear series of assumed functions:

$$
W(x, y)=\sum_{m=1}^{p} \sum_{n=1}^{q} A_{m n} X_{m}(x) Y_{n}(y)
$$

The assumed functions $X_{m}(x)$ and $Y_{n}(y)$ must be admissible, such that they satisfy the essential boundary conditions of the plate. The characteristic equations of vibrating beams are used as the assumed functions $X_{m}$ and $Y_{n}$. The characteristic equations of vibrating beams are chosen so that the boundary conditions of the beam matches those of the plate, guaranteeing satisfaction of the essential boundary conditions. The method of determining the set of characteristic functions and a summary of the properties of these functions were given by Young. ${ }^{14}$ The characteristic functions $X_{m}(x)$ for the free-free beam used in this study are given as follows:
Table 1 Material constants of the tested plates

\begin{tabular}{lccccc}
\hline \hline Material & $E_{1}, \mathrm{GPa}$ & $E_{2}, \mathrm{GPa}$ & $G_{12}, \mathrm{GPa}$ & $\nu_{12}$ & $\rho, \mathrm{kg} / \mathrm{m}^{3}$ \\
\hline Graphite/epoxy & 139.3 & 9.72 & 5.586 & 0.29 & 1550 \\
Aluminum & 70 & 70 & 26 & 0.33 & 2700 \\
\hline
\end{tabular}

Table 2 Dimensions of the tested plates

\begin{tabular}{lccc}
\hline \hline Tested plate & $a, \mathrm{~mm}$ & $b, \mathrm{~mm}$ & $h, \mathrm{~mm}$ \\
\hline Aluminum square plate & 80 & 80 & 1 \\
{$[0]_{16}$ Square plate } & 99 & 99 & 2 \\
{$[0]_{16}$ Rectangular plate } & 99 & 52 & 2 \\
{$[0 / 45 / 90 /-45]_{2 s}$} & 102 & 102 & 2 \\
$\quad$ Square plate & 102 & 51 & 2 \\
$\begin{array}{l}{[0 / 45 / 90 /-45]_{2 s}} \\
\quad \text { Rectangular plate }\end{array}$ & & & \\
\hline \hline
\end{tabular}

$$
\begin{gathered}
X_{1}(x)=1 \\
X_{2}(x)=\sqrt{3}[1-(2 x / \ell)] \\
X_{m}(x)=\cosh \left(c_{m} x / \ell\right)+\cos \left(c_{m} x / \ell\right) \\
-\alpha_{m}\left[\sinh \left(c_{m} x / \ell\right)+\sin \left(c_{m} x / \ell\right)\right], \quad m=3,4,5, \ldots
\end{gathered}
$$

where $\ell$ is the length of the beam. The numerical values of $c_{m}$ and $\alpha_{m}$ are given by Young. ${ }^{14}$ The functions $X_{1}(x)$ and $X_{2}(x)$ represent a rigid-body translation and rotation and are included to obtain a complete orthogonal set. Similar expressions also are used for $Y_{n}(y)$. By using the stationary potential energy theory, the Rayleigh-Ritz technique will provide a discrete number of stationary values $\omega_{s}$, which are the lowest upper bounds of the actual natural frequencies. ${ }^{15}$

A T300/976 graphite/epoxy composite plate with stacking sequence $[0 / 45 / 90 /-45]_{2 s}$ and $[0]_{16}$ and an isotropicaluminum 6061 T6 plate are used in this study. The material properties of the graphite/epoxy lamina and the aluminum are indicated in Table 1, where $E_{1}$ and $E_{2}$ are Young's moduli in the longitudinal and transverse directions, respectively; $G_{12}$ is in-plane shear modulus; and $v_{12}$ is the Poisson's ratio of the lamina. The averaging process is performed as follows: 1) Form the stiffness coefficients for the lamina; 2) use tensor transformation for each lamina to obtain transformed lamina stiffness matrice for different fiber orientations; 3 ) construct the laminate bending stiffness; and 4) finally determine the effective laminate engineering constants from Eqs. (9-12). The effective laminate engineering constants for $[0]_{16}$ and $[0 / 45 / 90 /-45]_{2 s}$ laminate plates are obtained from the material properties of lamina shown in Table 1, and the results for $[0]_{16}$ are

$$
E_{x}=139.3, \quad E_{y}=9.72, \quad G_{x y}=5.586, \quad v_{x y}=0.29
$$

and for $[0 / 45 / 90 /-45]_{2 s}$ are

$$
\begin{aligned}
E_{x} & =70.79, & E_{y} & =44.749 \\
G_{x y} & =17.195, & v_{x y} & =0.288
\end{aligned}
$$

where $E_{x}, E_{y}$, and $G_{x y}$ are in gigapascals. The dimensions of the tested plates are shown in Table 2.

\section{Experimental Setup and Inverse Evaluation of Material Constants}

To increase the intensity of light reflection of the specimens and the contrast of fringe patterns, the surfaces of plates are coated with white paint, which is mixed with fine powder. The self-arranged AF-ESPI system, as shown in Fig. 1, is employed to perform the out-of-plane vibration measurement. A He-Ne laser with $30 \mathrm{~mW}$ and wavelength $\lambda=632.8 \mathrm{~nm}$ is used as the coherent light source. The laser beam is divided into two parts, the reference and object beams, by a beamsplitter. The object beam travels to the specimen and then reflects to the CCD camera. The reference beam is directed to the CCD camera via the mirror and reference plate. Note that the optical path and light intensity of these two beams remain identical in the experimental setup. The plate is excited by the piezostack actuator (Physik Instrumente; $5 \times 5 \times 10 \mathrm{~mm}$ ), which is attached to the specimen. The piezoelectric actuator is usually attached in the 
center of the opposite face of the specimen. However, if the nodal lines pass the center of the specimen, then the piezoelectric actuator will be moved to other location. To achieve the sinusoidal output, a function generator HP33120A (Hewlett Packard) connected to a 4005 power amplifier (NF Electronic Instruments) is used.

A plate with all edges free is used to have the ideal boundary conditions for theoretical and experimental simulations. The resonant frequency and the correspondentmode shape for the vibrating plate are determined experimentally by using the noncontacting optical method of AF-FSPI. The first few resonant frequencies determined experimentally are used for inverse evaluation of the material properties of the tested plate by the Rayleigh-Ritz technique and the Simplex method ${ }^{16}$ for error function minimization. An error function is introduced to quantify the difference between the inversed value and the experimental result:

$$
E=\sum_{i=1}^{N}\left(\frac{\omega_{i}-\omega_{i}^{\exp }}{\omega_{i}^{\exp }}\right)^{2}
$$

where $N$ is the total number of the resonant frequencies to be used for inversed calculation, $\omega_{i}$ is the reconstructed resonant frequency obtained from the inversed value of material constants, and $\omega_{i}^{\exp }$ is the experimental measurement of resonant frequency by AF-ESPI. The material constants optimally searched must be such that the error function $E$ is the global minimum value. The convergence criterion used for the Simplex algorithm is that the difference of the material constants obtained in the two iterations is less than $0.01 \%$. After the material constants have been determined, a direct numerical calculation is performed by the finite element method to obtain the vibrating mode shapes. The numerical calculations are carried out by using the finite element package ABAQUS ${ }^{17}$ in which a nine-node,two-dimensionalshell element (S9R5) and five degrees of freedom at each node are selected to analyze the problem.

To test the analyzed procedure, an isotropic aluminum square plate is used before the investigation for composite plates. The material properties and dimensions of the aluminum plate are listed in Tables 1 and 2. Five initial guesses (Table 3) for different mate-
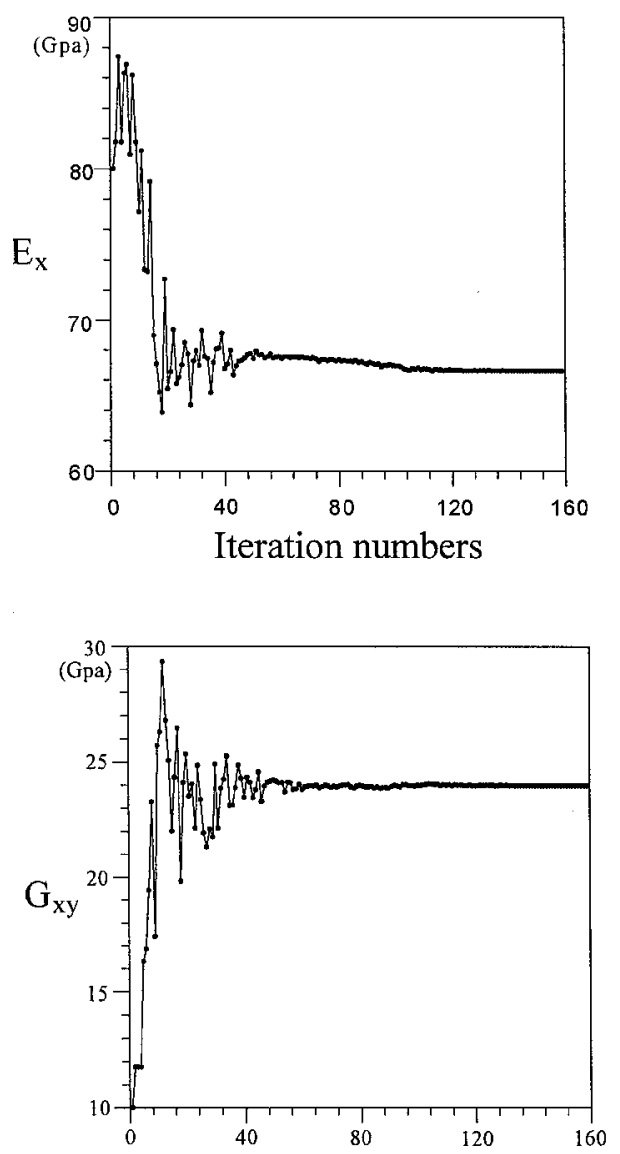

Iteration numbers rial combinations are performed for the inverse calculations. These values are used in a Fortran program in which equations derived by the Rayleigh-Ritz technique are used to determine the resonant frequencies. The iteration procedure is performed by using the Simplex algorithms until convergence values of the material constants are obtained. The five different initial guesses are converged to almost the same result, as indicated in Table 4. This shows the good convergence characteristics using the Simplex algorithm. The iteration numbers are from 90 to 175 , which depend on the value of initial guess and the convergence criterion, and the CPU times are all within $10 \mathrm{~s}$ using a personal computer (Intel Pentium $133 \mathrm{MHz}$ ). The iteration and convergence of the material constants for the fifth initial guess are shown in Fig. 2. The resonant

Table 3 Initial guesses of material constants for the aluminum plate

\begin{tabular}{lcccc}
\hline \hline $\begin{array}{l}\text { Initial guess } \\
\text { number }\end{array}$ & $\begin{array}{c}E_{1}, \\
\mathrm{GPa}\end{array}$ & $\begin{array}{c}E_{2}, \\
\mathrm{GPa}\end{array}$ & $\begin{array}{c}G_{12}, \\
\mathrm{GPa}\end{array}$ & $\nu_{12}$ \\
\hline 1 & 70 & 70 & 26 & 0.33 \\
2 & 30 & 30 & 12 & 0.2 \\
3 & 95 & 95 & 34 & 0.4 \\
4 & 40 & 40 & 40 & 0.5 \\
5 & 80 & 50 & 10 & 0.6 \\
\hline \hline
\end{tabular}

Table 4 Inverse evaluations of material constants for five different initial guesses in Table 3 and the iteration number

\begin{tabular}{lccccc}
\hline \hline Converge value & $\begin{array}{c}E_{1}, \\
\mathrm{GPa}\end{array}$ & $\begin{array}{c}E_{2}, \\
\mathrm{GPa}\end{array}$ & $\begin{array}{c}G_{12}, \\
\mathrm{GPa}\end{array}$ & $v_{12}$ & $\begin{array}{c}\text { Iteration } \\
\text { number }\end{array}$ \\
\hline 1 & 66.614 & 66.605 & 23.979 & 0.2852 & 90 \\
2 & 66.608 & 66.601 & 23.979 & 0.2854 & 129 \\
3 & 66.620 & 66.613 & 23.977 & 0.2852 & 175 \\
4 & 66.614 & 66.602 & 23.979 & 0.2854 & 121 \\
5 & 66.613 & 66.612 & 23.978 & 0.2852 & 158 \\
Theoretical value & 70 & 70 & 26 & 0.33 & - \\
Difference, \% & -4.84 & -4.85 & -7.78 & -13.52 & - \\
\hline \hline
\end{tabular}
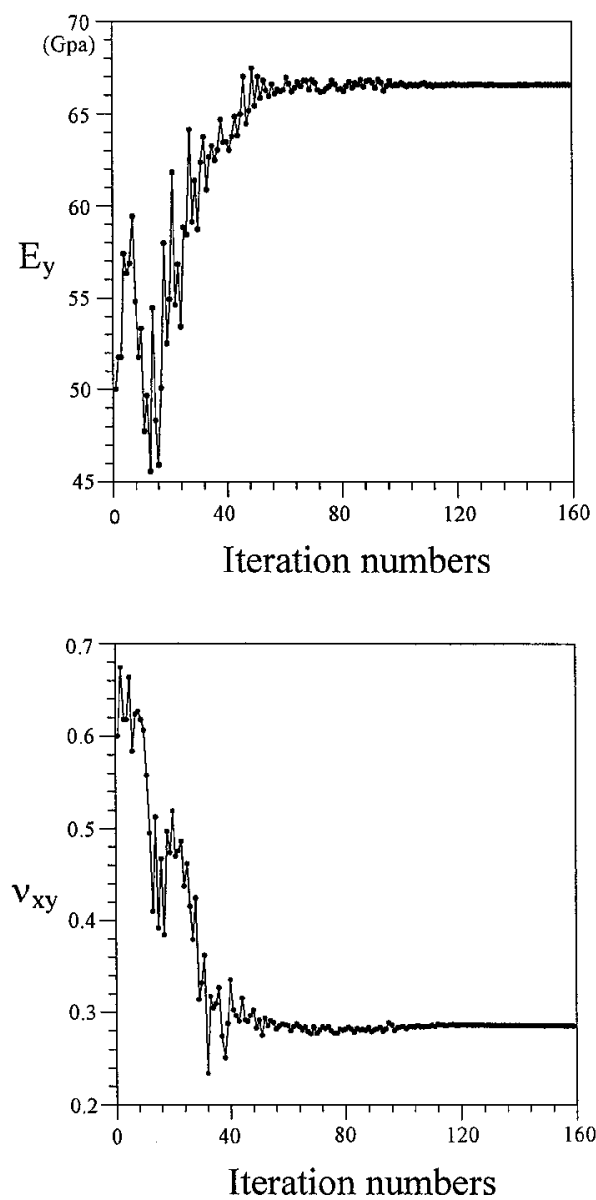

Fig. 2 Iteration and convergence of material constants for the fifth initial guess of the aluminum plate. 


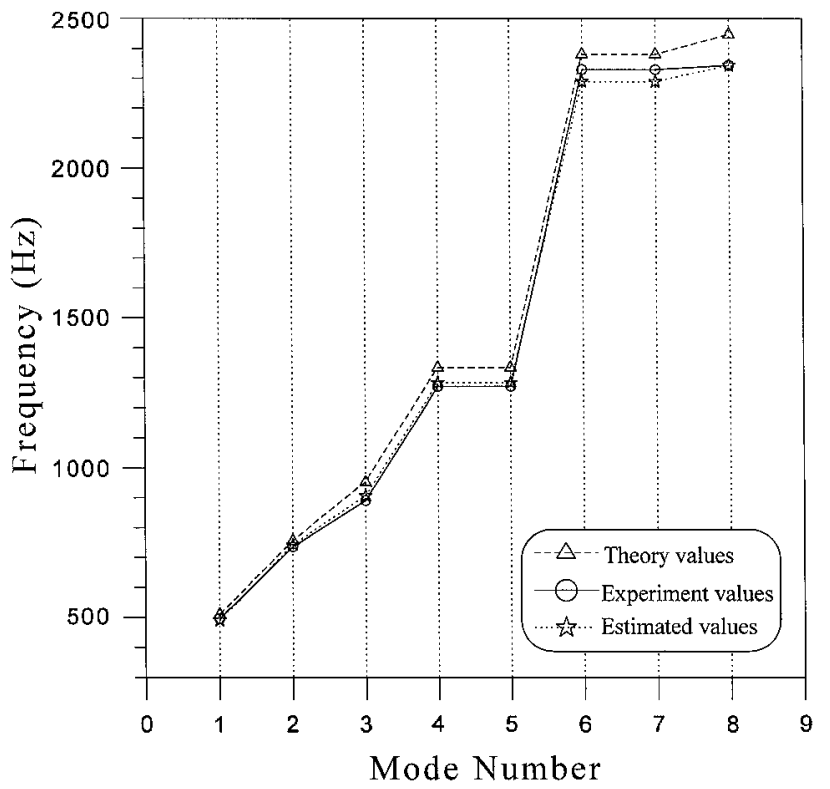

Fig. 3 First eight resonant frequencies from the experimental measurement and the calculated value for the aluminum plate.

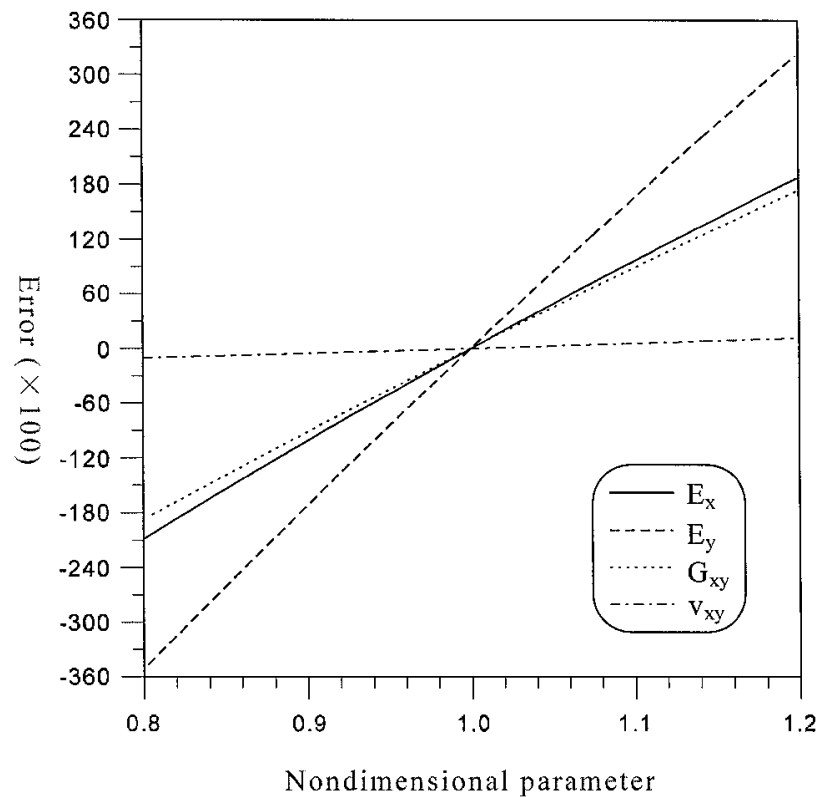

Fig. 4 Plot of error functions for material constants $E_{x}, E_{y}, G_{x y}$, and $\nu_{x y}$ for $[0]_{16}$ composite plate.

frequencies for the experimental measurement and the calculated value from the inverse evaluation material constants are shown in Fig. 3. We can see that the inverse estimated values of the resonant frequencies agree very well with the experimental measured results for the eight modes. This also shows the excellent reliability and convergence of the Simplex algorithms for inverse evaluations of the material constants by using resonant frequencies.

Before inverse evaluating the material constants for composite plates, we will discuss qualitatively the error of the resonant frequencies that are induced by each individual inversed material constant. The results for the $[0]_{16}$ composite plate are shown in Fig. 4, in which the nondimensionalparameter equal to 1 is the exact value. We can see clearly that a large variation of $v_{x y}$ from the exact value will cause only a very small error of resonant frequencies. This indicates that the parameter $v_{x y}$ is insensitive to the resonant frequencies, and we will expect that the inverse value of $v_{x y}$ will not have accuracy similar to $E_{x}, E_{y}$, and $G_{x y}$.

Finally, the composite square and rectangular plates with stacking sequence $[0]_{16}$ and $[0 / 45 / 90 /-45]_{2 s}$ are investigated, and the
Table 5 Inverse evaluations of material constants for $[0]_{16}$ square and rectangular composite plates

\begin{tabular}{lcccc}
\hline \hline$[0]_{16}$ & $E_{x}, \mathrm{GPa}$ & $E_{y}, \mathrm{GPa}$ & $G_{x y}, \mathrm{GPa}$ & $v_{x y}$ \\
\hline Theoretical value & 139.3 & 9.72 & 5.586 & 0.29 \\
Inverse value & 134.58 & 9.322 & 5.454 & 0.334 \\
$\quad$ (square plate) & & & & \\
Difference, \% & -3.39 & -4.09 & -2.37 & 15.10 \\
$\quad$ Inverse value & 137.04 & 9.909 & 5.241 & 0.248 \\
$\quad$ (rectangular plate) & & & & \\
Difference, \% & -1.63 & -1.94 & -6.17 & -14.6 \\
\hline \hline
\end{tabular}

Table 6 Inverse evaluations of material constants for $[0 / 45 / 90 /-45]_{2 s}$ square and rectangular composite plates

\begin{tabular}{lcccc}
\hline \hline$[0 / 45 / 90 /-45]_{2 s}$ & $E_{x}, \mathrm{GPa}$ & $E_{y}, \mathrm{GPa}$ & $G_{x y}, \mathrm{GPa}$ & $v_{x y}$ \\
\hline Theoretical value & 70.79 & 44.749 & 17.195 & 0.288 \\
Inverse value & 66.387 & 41.057 & 16.125 & 0.245 \\
$\quad$ (square plate) & & & & \\
$\begin{array}{l}\text { Difference, \% } \\
\text { Inverse value }\end{array}$ & -6.22 & -8.25 & -6.22 & -14.86 \\
$\quad$ (rectangular plate) & 65.928 & 43.103 & 15.875 & 0.332 \\
Difference, \% & 6.87 & 3.68 & 7.68 & 15.28 \\
\hline \hline
\end{tabular}

inverse evaluation of material constants based on the resonant frequencies obtained from experimental measurements are shown in Tables 5 and 6 . The procedure for inverse evaluation of composite plates is the same as that used for the isotropic aluminum plate. The iteration and convergence characteristics are very similar to that of aluminum plate, and the CPU time for the inverse calculation is about $10 \mathrm{~s}$ using a personal computer. The difference of $E_{x}, E_{y}$, and $G_{x y}$ is below $6 \%$ for the $[0]_{16}$ plate and is below $8 \%$ for the $[0 / 45 / 90 /-45]_{2 s}$ plate, but the difference of $v_{x y}$ is about $15 \%$ for both the $[0]_{16}$ and $[0 / 45 / 90 /-45]_{2 s}$ plates. The difference of $v_{x y}$ for the inverse value and the theoretical result is the largest one, as expected. The larger error in the $v_{x y}$ measurement than that in $E_{x}$, $E_{y}$, and $G_{x y}$ is consistent with the sensitivity of $v_{x y}$ being the smallest one. Note that the theoretical values are the effective laminate engineering constants, as discussed in the preceding section. Hence, the difference between the inverse value and the theoretical result is mainly due to the errors induced by measuring the resonant frequencies from the AF-ESPI technique, the accuracy of the Rayleigh-Ritz method, and the suitability of the method for determination of the effective laminate engineering constants from the material properties of the lamina. From the obtained inverse evaluation of the material constants, the first eight mode shapes are computed by ABAQUS, and the results are compared with the experimentalobservationfrom AF-ESPI, as shown in Fig. 5. The vibrating displacement of the composite plate (solid lines) given by the numerical analysis is in the opposite direction to that with the dotted lines. The transition from solid lines to dotted lines corresponds to a zero displacement line, or a nodal line. The brightest fringes of the experimentalresults represent the nodal lines of the vibrating plate at resonant frequencies. The rest of the fringes are contours of constant displacement. It can be seen that the mode shapes obtained experimentally are in good agreement with those obtained numerically. We can see from Fig. 5 that there is no mismatch or incorrect correlation of the experimental and numerical mode shapes; hence, we have the confidence that the resonant frequencies determined in the experimental measurement are correct. Note that, if there are resonant frequencies that are either missing or are not in order in the experimental measurement, then large errors will be induced in evaluating the material constants. However, because we can obtain the resonant frequency and mode shape experimentally at the same time, the resonant frequency is used for the inversed evaluation of the material constants, and the correspondent mode shape can be used as a check of the result to ensure that the missing or the mismatch of the resonant frequency will not occur. The optical AF-ESPI measuring system and the inversed algorithms used provide a reliable and self-consistent methodology for evaluating the material constants of composite plates. 

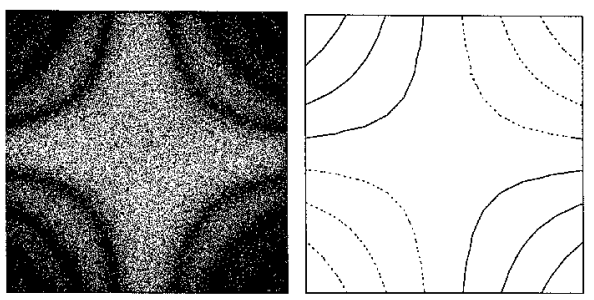

Mode1 $(2,2)$
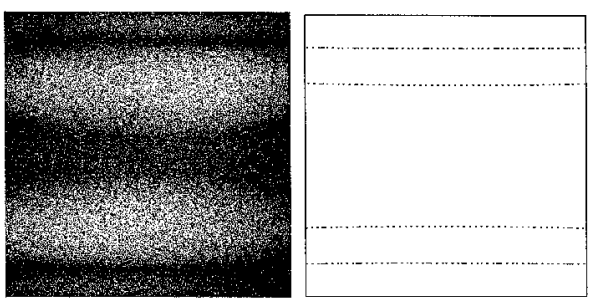

Mode2 (1,3)
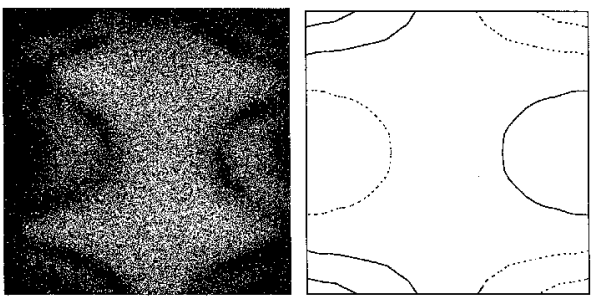

Mode3 $(2,3)$
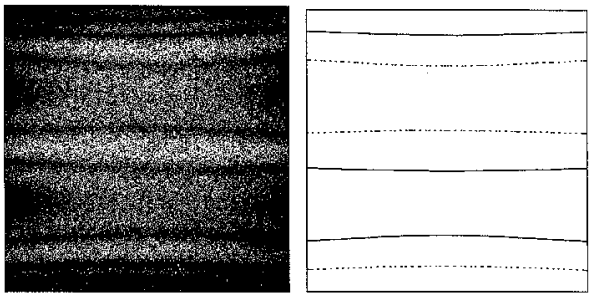

Mode4 $(1,4)$
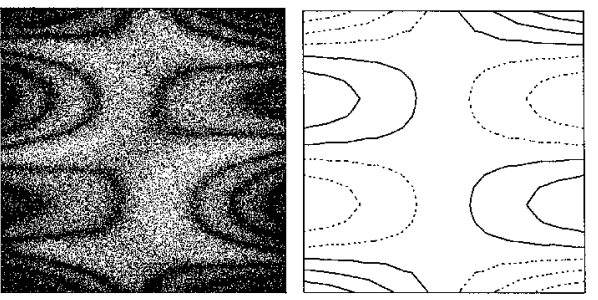

Mode5 $(2,4)$
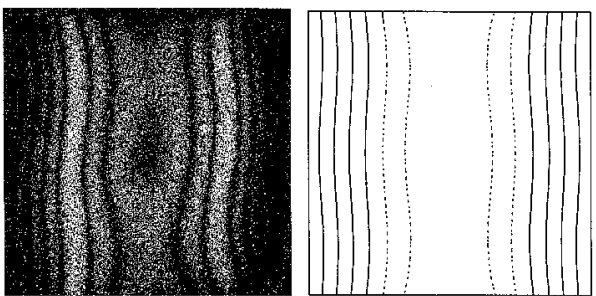

Mode6 $(3,1)$
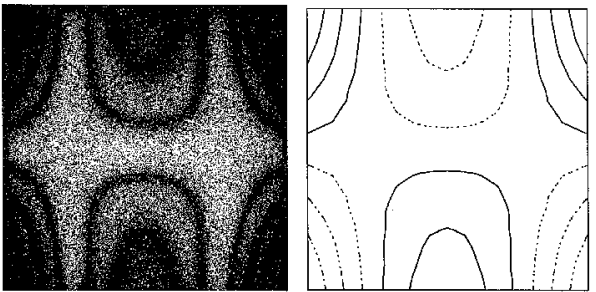

Mode $7(3,2)$
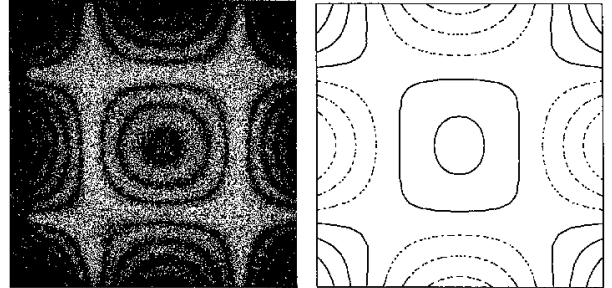

Mode8 $(3,3)$

Fig. 5 First eight mode shapes for $[0]_{16}$ composite square plate obtained from the experimental observation and numerical calculation.

\section{Conclusions}

Investigation of the vibration problem by employing the ESPI method has the advantages of real-time and noncontact measurement, submicrometer sensitivity, digital image processing, etc. In this paper, a self-arranged AF-ESPI optical setup with good visibility and noise reduction has been established to obtain experimentally the resonant frequencies and the corresponding mode shapes of plates at the same time. A computer program in which equations derived by the Rayleigh-Ritz technique is developed to determine the resonant frequencies of vibrations for composite plates with a tractionfree boundary condition. The Simplex iteration algorithms are incorporated into the computer program for the inverse evaluation of two Young's moduli, the in-plane shear modulus, and a Poisson ratio for composite plates. The difference of $E_{x}, E_{y}$, and $G_{x y}$ is below $8 \%$, and that of $v_{x y}$ is below $15 \%$ when compared with the predicted results for the effective laminate engineering constants that are evaluated from the material properties of lamina. The difference between the inverse value and the theoreticalpredictedresults is mainly due to the errors that are induced by measuring the resonant frequencies from the AF-ESPI technique, the accuracy of the Rayleigh-Ritz method, and the suitability of the method for determination of the effective laminate engineering constants. The potential time savings and versatility of the technique, combined with several promising results, indicate that the method warrants further consid- eration. With continued development, this technique for obtaining material constants will become a valuable nondestructive test.

\section{Acknowledgment}

The authors gratefully acknowledge the financial support of this research by the National Science Council (Republic of China) under Grant NSC 87-2212-E002-036.

\section{References}

${ }^{1}$ Rastogi, P. K., Holographic Interferometry, Springer-Verlag, Berlin 1994.

${ }^{2}$ Butters, J. N., and Leendertz, J. A., "Speckle Pattern and Holographic Techniques in Engineering Metrology," Optics Laser Technology, Vol. 3, No. 1, 1971, pp. 26-30.

${ }^{3}$ Jones, R., and Wykes, C., Holographicand Speckle Interferometry, Cambridge Univ. Press, Cambridge, England, UK, 1989, pp. 165-196.

${ }^{4}$ L $\varnothing \mathrm{kberg}$, O. J., and Hogmoen, K., "Use of Modulated Reference Wave in Electronic Speckle Pattern Interferometry,” Journal of Physics E: Scientific Instruments, Vol. 9, 1976, pp. 847-851.

${ }^{5}$ Creath, K., and Slettemoen, G. A., "Vibration-Observation Techniques for Digital Speckle-Pattern Interferometry," Journal of the Optical Society of America A, Vol. 2, No. 10, 1985, pp. 1629-1636.

${ }^{6}$ Pouet, B., Chatters, T., and Krishnaswamy, S., "Synchronized Reference Updating Technique for Electronic Speckle Interferometry," Journal of Nondestructive Evaluation, Vol. 12, No. 2, 1993, pp. 133-138. 
${ }^{7}$ Wang, W. C., Hwang, C. H., and Lin, S. Y., "Vibration Measurement by the Time-Averaged Electronic Speckle Pattern Interferometry Methods," Applied Optics, Vol. 35, No. 22, 1996, pp. 4502-4509.

${ }^{8} \mathrm{Ma}, \mathrm{C}$. C., and Huang, C. H., "The Investigation of Three-Dimensional Vibration for Piezoelectric Rectangular Parallelepipeds by Using the AF-ESPI Method," International Journal of Solids and Structures (submitted for publication).

${ }^{9}$ Huang, C. H., and Ma, C. C., "Vibration Characteristics for Piezoelectric Cylinders Using Amplitude-Fluctuation Electronic Speckle Pattern Interferometry," AIAA Journal, Vol. 36, No. 12, 1998, pp. 2262-2268.

${ }^{10}$ Zelenev, Y. V., and Electrova, L. M., "Determination of the Dynamic Parameters of Polymer Plates," Soviet Physics-Acoustics, Vol. 18, No. 3, 1973, pp. 339-341.

${ }^{11}$ Wolf, J. A., and Carne, T. G., "Identification of the Elastic Constants for Composites Using Modal Analysis," Society for Experimental Stress Analysis, Paper A-48, San Francisco, CA, 1979.
${ }^{12}$ Caldersmith, G., and Rossing, T. D., "Determination of Modal Coupling in Vibrating Rectangular Plates," Applied Acoustics, Vol. 17, No. 1, 1984, pp. 33-44.

${ }^{13}$ Deobald, L. R., and Gibson, R. F., "Determination of Elastic Constants of Orthotropic Plates by a Modal Analysis/Rayleigh-Ritz Technique," Journal of Sound and Vibration, Vol. 124, No. 2, 1988, pp. 269-283.

${ }^{14}$ Young, D., "Vibration of Rectangular Plates by the Ritz Method," Journal of Applied Mechanics, Vol. 17, No. 4, 1950, pp. 448-453.

${ }^{15}$ Leissa, A. W., "The Free Vibration of Rectangular Plates," Journal of Sound and Vibration, Vol. 31, No. 3, 1973, pp. 257-293.

${ }^{16}$ Nelder, J. A., and Mead, R., "A Simplex Method for Function Minimization," Computer Journal, Vol. 7, 1965, pp. 308-313.

17 “ABAQUS User's Manual,” Version 5.5, Hibbit, Karlsson, and Sorensen, Inc., Pawtucket, RI, 1995.

A. Berman Associate Editor 